\title{
Efficacy of air purifier therapy in allergic rhinitis
}

\author{
Luo Jia-ying, ${ }^{1}$ Chen Zhao, ${ }^{1}$ Guo Jia-jun, ${ }^{1}$ Guo Zi-jun, ${ }^{1}$ Lan Xiao, ${ }^{1}$ Sun Bao-qing ${ }^{1}$
}

\begin{abstract}
Background: With the rising prevalence of allergic rhinitis, the utility of indoor environmental management deserves increasing scrutiny. This research aims at evaluating the ability of air purifiers to be a therapy of allergic rhinitis.

Methods: 32 subjects $(25 \pm 13.5$ years old) diagnosed with allergic rhinitis were selected and HEPA air purifiers placed in their bedrooms for 4 months. Before the intervention and each month, dust samples were collected with a vacuum cleaner and the dust collector assessed for allergen content. Additionally, static dust collectors were left in place all month to collect dust by sedimentation. Particulate matter (PM) was assessed in terms of $\mathrm{PM}_{\text {indoor/outdoor }}$ ratios. The Rhinitis Quality of Life Questionnaire (RQLQ) was used to assess symptoms.
\end{abstract}

Results: Der p $1(78(30,82)$ ng/g) was the dominant dust mite allergen in air samples of patients' bedroom as well as static collections. Der f1 $(444(345,667) \mathrm{ng} / \mathrm{g})$ was the dominant allergen in bedding. Der f1 levels in both air and bed sampling significantly decreased after initiation of HEPA air purifiers $(P<0.05) . P 1_{1} 0_{\text {indoor/outdoor }}, \mathrm{PM} 2.5_{\text {indoor/outdoor }}$ PM10 ${ }_{\text {indoor/outdoor }}$ all decreased $(P<0.001)$ with the HEPA filtration intervention. According to RQLQ data, HEPA filtration was associated with improvements in activity limitation, non-nasal-eye symptoms, practical problems, and nasal symptoms $(P<0.001)$.

Conclusion: HEPA air purifiers can effectively reduce PM and HDM allergen concentration in the indoor air, and thereby improve clinical manifestations of patients with AR.

Keywords: Allergic rhinitis, Air purifier, House dust mite, Rhinitis Quality of Life Questionnaire, PM

\footnotetext{
From:

${ }^{1}$ State Key Laboratory of Respiratory Disease, National Clinical Center for Respiratory Diseases, Guangzhou Institute of Respiratory Health, First Affiliated Hospital of Guangzhou Medical University
}

Corresponding author:

Sun Bao-qing

151 Yan Jiang Rd. GuangZhou(Canton).510000. China

Email: sunbaoqing@vip.163.com

\section{Introduction}

Allergic rhinitis is the most common type of rhinitis and its burden is increasing globally. ${ }^{1,2}$ It affects quality of life and can be expensive. Wang et $\mathrm{al}^{3}$ illustrated increasing prevalence of allergic rhinitis in the major cities in China. The total prevalence has increased from $11.1 \%$ in 2005 to $17.7 \%$ in 2011 while Guangzhou's prevalence has increased from $13.2 \%$ to $17.4 \%$. Genetic variance cannot cause this pace of change; either some environmental factor is causative, or perhaps a disease classification factor is relevant (diagnostic shift from non-specific rhinitis, or increased awareness of allergic disease by the population). Importantly, environment allergens have well-elucidated impacts on allergic rhinitis. ${ }^{2}$

Ambient particulate air pollution (often represented as particulate matter, PM) is a risk factor for allergic disease. ${ }^{2}$ Outdoor PM may contain various metallic elements that initiate or augment pediatric allergic rhinitis. ${ }^{4}$ But the exposure to indoor ambient allergens is of primary importance. ${ }^{5}$ For allergic patients, therapeutic interventions are often undertaken to help minimize relevant allergen exposure. ${ }^{6}$

Based on prior research, it is assumed that these environmental modification efforts should be effective at improving the quality of life of allergic rhinitis patients, by means of decreasing the ambient concentration of PM and relevant allergens. Although HEPA air purifiers are presented as tools to filter PM and allergens from air that passes through the device, the clinical utility of these units remain uncertain. We performed an intervention with air purifiers to determine the effect on improving the indoor environment, while simultaneously evaluating the intervention's effect as an adjuvant therapy.

\section{Methods \\ Ethical Statement}

The study protocol was approved by the Ethics Committee of First Affiliated Hospital, Guangzhou Medical University (No. GYFYY-2015-47). Written informed consent was obtained from 
parents or guardians of all children before they participated in this study.

\section{Rhinitis Quality of Life Questionnaires}

The Rhinitis Quality of Life Questionnaire (RQLQ) was designed by Juniper and others. ${ }^{7}$ The RQLQ used in this study was based on Juniper's RQLQ with slight modifications. This RQLQ evaluates limitation of activity, sleep (lack of a good night's sleep, waking during the night, difficulty getting to sleep), non-nasal symptoms (tiredness, fatigue, worn out, reduced productivity, poor concentration, thirst), practical problems related to allergies (need to blow nose repeatedly, need to rub/eyes, inconvenience of having to carry tissues or handkerchief), nasal symptoms (stuffy/blocked, sneezing, runny, itchy), eye symptoms (itchy, watery, swollen, sore), emotions (irritable, frustrated, impatient, embarrassed by nose/eye symptoms, restless). There are 28 questions requiring answers on a $0-6$ points scale. Higher scores in RQLQ indicate more adverse effects on the quality of life.

\section{Screening Method}

Subjects diagnosed with allergic rhinitis who presented to the First Affiliated Hospital of Guangzhou Medical University were recruited. Our diagnoses were based on a 2015 allergic rhinitis clinical practice guideline. ${ }^{8}$ The patients underwent allergen skin prick testing. Eligibility for the study required positive skin prick test positivity to at least one of the house dust mite (HDM) primary allergens, Der p 1 or Der f1, or alternatively, positive ImmunoCAP testing of serum for Der p 1 specific IgE and/or Der f1 specific IgE (defined as $\geq 0.35 \mathrm{kU} / \mathrm{L}$ ). The subjects were not able to change their accommodations during the study. Furthermore, study participants needed to be able to properly cooperate with our research (including correct and persistent use of the supplied air purifier, performing the questionnaire investigations, completing follow up visits, cooperating with sample collecting). The details of the research were provided and all subjects signed informed consent.

\section{Intervention}

This study provided a new HEPA air purifier (BA1030/1045, BRI Air Purifier, Xiamen, China, CADR: $200-400 \mathrm{~m}^{3} / \mathrm{h}$ ) for use from October, 2015 to February, 2016. Our team taught subjects or their parents how to correctly use the air purifier and confirmed correct usage during each return visit.

\section{The Use of Air Purifiers}

Air purifiers were set near the head of the bed while avoiding blockage by foreign matter (such as clothes). No concurrent humidifiers were allowed and it was recommend that door and windows should be kept closed when patients were utilizing the air purifier.

\section{Review Methods}

Trained investigators performed phone interviews to investigate the subjects' compliance with air purifier use each month. This involved ascertaining whether they correctly used the air purifier, how many times they used it in a week, and how long they used it each time. In the event incorrect usage was identified, education was provided.

\section{Sample Collection}

A trained investigator was assigned to collect the sample and data from each subject's room, using consistent technique throughout the study.

\section{Air dust sample collection}

Air dust samples were collected every month in the subjects' homes. A vacuum dust sampler (FCD-50 double dust sampler, China Galaxy Science Co. Ltd, Yancheng, Jiangsu, China) was set in the middle of the subject's room. The dust sampler utilized glass fiber filtering (A/E glass fiber filter, America PALL company, ply: $1 \mu \mathrm{m}$, diameter: $47 \mathrm{~mm}$ ). Airflow volume was adjusted to $20 \mathrm{l} / \mathrm{min}$. The dust sampling occurred for 4 hours, during which time doors and windows remained sealed closed.

\section{Bedding dust sample collecting}

Simultaneously, bedding dust samples were collected by means of filter paper glass fiber and a vacuum cleaner (Hazier ZW1401B). An elastic band was used to hold the filter on the top of the vacuum hose. Bedding dust samples were collected over $15 \mathrm{~min}$.

\section{Static dust sample collection}

A glass fiber filter was placed within a $60 \mathrm{~mm}$ round open culture plate in each subject's bedroom to collect HDM by natural sedimentation. Each sample collection occurred for one month, during which time the sampling material was not moved or covered.

\section{PM data collection}

Indoor and outdoor $\operatorname{PM}(1.0,2.5,10)$ concentration data were collected by a DT-9881 Air Quality Detector (CEM) once a month. Indoor collections were performed at five locations (four corners of the bedroom and one point in the middle of the room). Outdoor collections were performed in triplicate on the balcony or out of the window.

\section{$R Q L Q$ data collection}

Each subject provided RQLQ questionnaire responses each month.

\section{Sample Extraction}

Dust samples were weighed immediately after collection. The glass fiber filter was shredded manually and placed into a $10 \mathrm{ml}$ flask to which was added $1 \mathrm{ml}$ of $1 \%$ BSA and $0.05 \%$ Tween-20 PBST at $4^{\circ} \mathrm{C}$ and shaken overnight. The fluid was then extracted by centrifugation at $4^{\circ} \mathrm{C}, 3000 \mathrm{~g}$ at $30 \mathrm{~min}$, and supernatants stored at $-20^{\circ} \mathrm{C}$.

\section{Assay of HDM Allergen}

HDM allergens Der p 1 and Der $\mathrm{f} 1$ were assayed by double -antibody sandwich ELISA kits according to the manufacturers' instructions (Indoor Biotechnologies, Charlottesville, VA, USA). The mass of Der $\mathrm{p} 1$ and Der $\mathrm{fl}$ in each gram of dust was calculated. 


\section{Statistical Analysis}

SPSS 19.0 statistical software (SPSS Inc, Chicago, IL, USA) was applied to report and analyze our data, including descriptive statistics for the subjects' basic characteristic (gender, age and sIgE), the duration of use of the HEPA air purifier, the concentrations of HDM allergens, PM and RQLQ results. The concentration of HDM was assessed by the Kolmogorov-Smirnov test. If normally distributed, independent sample $t$ testing was employed. Abnormally distributed data were assessed by independent sample nonparametric tests. $\mathrm{PM}_{\text {indor/outoor }}$ ratios were compared to the baseline ratio prior to intervention. Repetitive measure analysis of variance (ANOVA) was used to compare the monthly data (concentration of HDM allergen, PM, RQLQ). $P<0.05$ was considered statistically significant.

\section{Results}

Baseline Characteristics of the Subjects

32 subjects were enrolled (4-61 yrs old) (Table 1).

Table 1. Baseline characteristics of the subjects

\begin{tabular}{lcccc}
\hline & & $\mathbf{n}$ & $\%$ & $\overline{\mathbf{x}} \pm \mathbf{s}$ \\
\hline Sex & male & 12 & 37.5 & \\
& female & 20 & 62.5 & \\
Age & & & & $25 \pm 13.5$ \\
& $<18$ & 12 & 37.5 & \\
& $\geq 18$ & 20 & 62.5 & \\
Der p1 sIgE/ KU/L & & 32 & & $52 \pm 38.8$ \\
Der f1 sIgE/ KU/L & & 32 & & $50 \pm 38.5$ \\
\hline
\end{tabular}

\section{Duration of HEPA Air Purifier Use}

The mean duration of daily HEPA air purifier use was 9.6 \pm 3.3 hours and fairly consistent during the course of the study. (Figure 1)

\section{Baseline HDM Allergen Concentration in Bedroom}

Bedroom air and static collections found mainly Der $\mathrm{p} \mathrm{1,}$ while bedding samples were dominated mainly by Der f1. The bed's Der f1 and Der p 1 concentrations were substantially higher than air's Der f1 and Der p 1 (see Table 2).

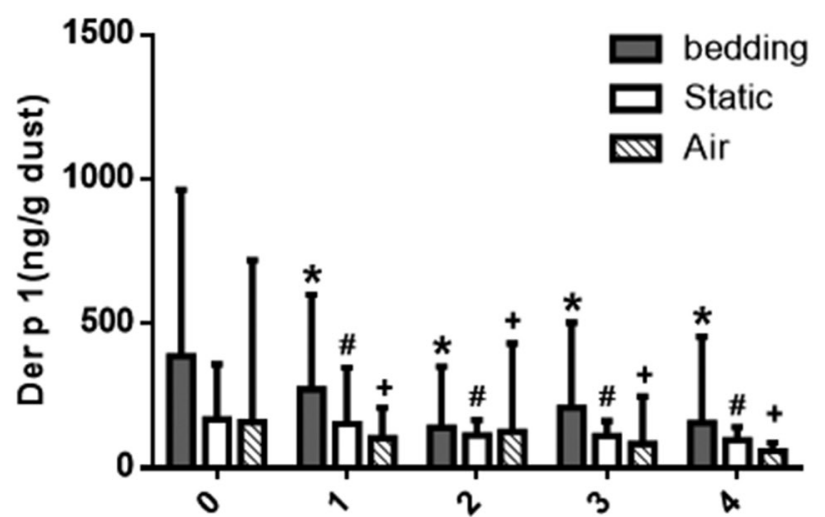

Figure 2. Changes in Der $p 1$ and Der $f 1$ concentrations.

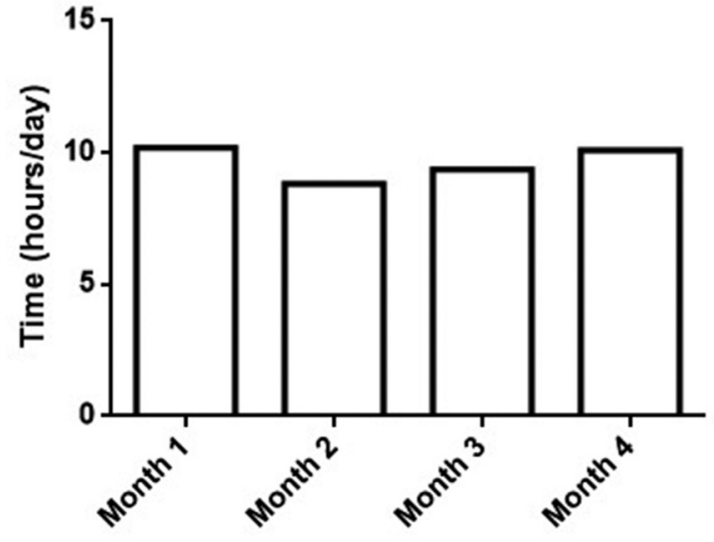

Figure 1. HEPA air purifier use by month.

Table2. Dust mite allergen levels before intervention

\begin{tabular}{lcc}
\hline & $\begin{array}{c}\text { Der } \mathbf{~} \mathbf{1} \\
\left(\mathbf{P}_{50}\left(\mathbf{P}_{25}, \mathbf{P}_{75}\right) \mathbf{n g} / \mathbf{g} \text { dust }\right)\end{array}$ & $\begin{array}{c}\text { Der } \mathbf{~ 1 ~} \\
\left(\mathbf{P}_{50}\left(\mathbf{P}_{25}, \mathbf{P}_{75}\right) \mathbf{n g} / \mathbf{g} \text { dust }\right)\end{array}$ \\
\hline air & $78(30,82)^{*}$ & $30(29,31)^{*}$ \\
bedding & $139(35,426)^{*}$ & $444(345,667)^{*}$ \\
static & $158(60,175)^{*}$ & $60(57,80)^{*}$ \\
\hline
\end{tabular}

${ }^{\star} P<0.05$

\section{Efficacy of Air Purifiers to Remove HDM Allergen}

Initiation of air purification was associated with significant declines in Der p 1 and Der f1 concentrations in static and bedding dust samples as shown in Figure 2. The concentration of house dust mite (HDM) in the air tended to decrease slightly with air purifier use, but the difference was not statistically significant. (Figure 2)

\section{Efficacy of Air Purifier on PM in Bedrooms}

PM1.0 indoor/outdoor' PM2.5 $_{\text {indoor/outdoor' }}$ PM10 ${ }_{\text {indoor/outdoor }}$ were found to be $0.94,0.89,0.91$, respectively, at baseline (prior to intervention). These ratios declined after initiation of the HEPA intervention, indicating relatively lower $\mathrm{PM}_{\text {indoors }}$ after initiating HEPA filtration (Figure 3). The changes in PM were also shown in Figure 3.

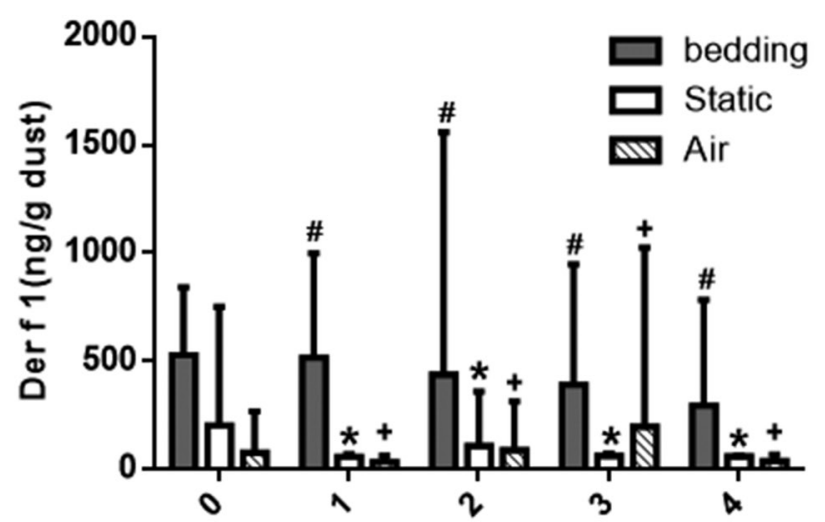


A

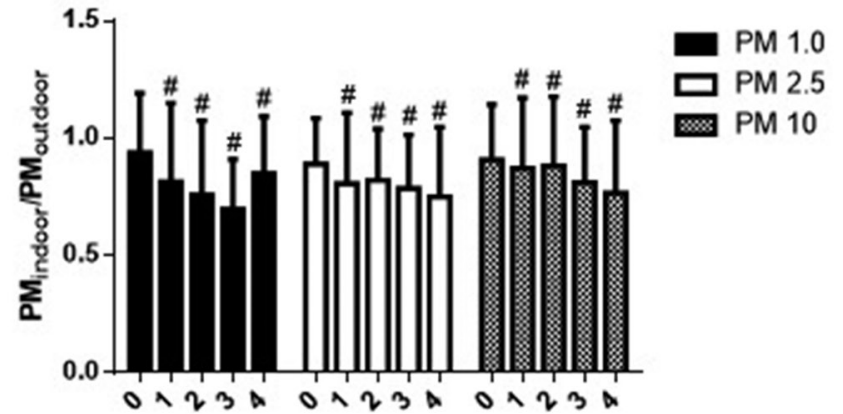

B

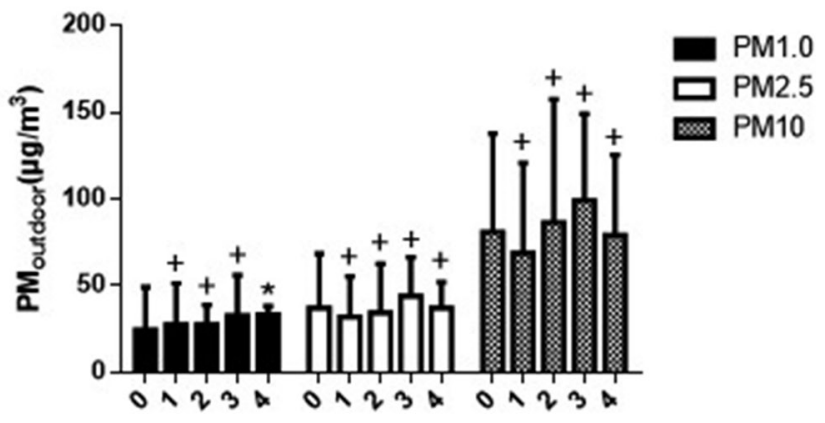

Figure 3. (A) Changes in $\mathbf{P M}$

; (B) Changes in $\mathrm{PM}_{\text {outdoor }}$

0: prior to intervention with HEPA filtration; 1: after 1 month; 2 : after 2 months; 3 : after 3 months; 4 : after 4 months. +: $P>0.05$, ${ }^{*}$ : $P<0.05$, \#: $P<0.001$. All compared with baseline.
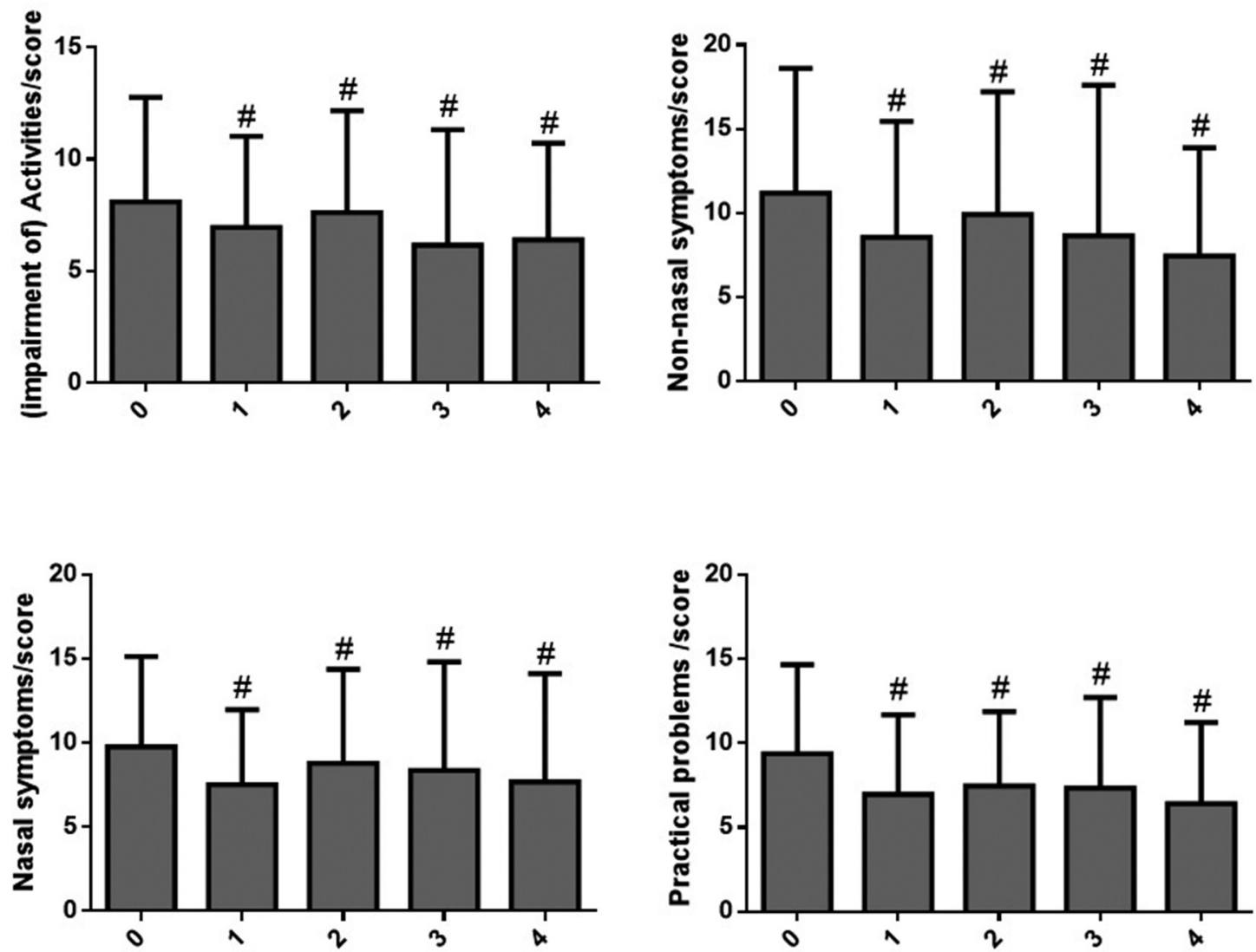

Figure 4. RQLQ score (Activity limitation, non-nasal symptoms, nasal symptoms, practical problems).

0: prior to intervention with HEPA filtration; 1: after 1 month; 2: after 2 months; 3: after 3 months; 4 : after 4 months. \#: P<0.001. All compared with baseline.

\section{Clinical Efficacy of Air Purifiers}

According to RQLQ questionnaire results, scores for limitation of activity, non hay-fever symptoms, practical problems, and nasal symptoms were significantly decreased after initiation of the air purifier (Figure 4). The scores from three other items (eye symptoms, emotional, and sleep) also tended to decrease, although not statistically significantly.

\section{Discussion}

HDM is an important cause of allergy symptoms, ${ }^{9}$ and $\mathrm{PM}$ serves as an allergic rhinitis risk factor. ${ }^{10}$ Interventions to decrease indoor HDM allergens and PM have been studied internationally. ${ }^{5}$ Studies incorporating air purifiers as an intervention remain few, and the evaluation of intervention effects on levels of HDM, PM, and patients' subjective symptoms are rarely reported in the literature.

According to our results, the concentration of Der f1 in bedding is significantly higher than that of Der $\mathrm{p} 1$ in our region during the seasons of this study. This result is consistent with the data of Wang et $\mathrm{al}^{11}$ The concentration of Der $\mathrm{p} 1$ in samples collected from air was relatively higher than the concentration in the bed sheets.

The concentrations of Der p 1 and Der f1 collected with the static method (constant collection over a full month) 
significantly decreased after initiating the air purifier. This is consistent with the data of Agrawal et al, ${ }^{12}$ which reveal that removal of dust mite from the air will diminish surface allergen by assuring that allergen sedimentation rate is decreased. It should be noted that our research used HEPA air filtration, while the one conducted by Agrawal et al ${ }^{12}$ used electrostatic air cleaners. Our research also found that the concentration of Der p 1 and Der f1 decreased in bedding. Surprisingly, the concentration of HDM allergen in air, as collected by ambient air dust sampler, did not decrease. It is possible that the airflow of the air sampling equipment stirred up static dust and that this affected the concentration of HDM allergen in the collected samples sufficiently to dampen the effect size of the air purifier on this variable. Overall, air purifiers as used in our study may effectively lessen indoor levels of HDM allergen.

Before utilizing the HEPA air purifier, both PM2.5 and $\mathrm{PM} 10_{\text {indoor/outdoor }}$ was significantly lower than 1 . Our data support the contention that indoor air is better than outdoor air in general. After the air purifier was used, the PM1.0

PM2.5 $5_{\text {indoor/outdoor }}$ and PM10 ${ }_{\text {indoor/outdoor }}$ all decreased, while there was no significant changes in the outdoor PM levels during the study period, which showed that the indoor PM levels were significantly decreased. This result is similar to Kajbafzadeh et al's results, ${ }^{13}$ which compared indoor PM to evaluate air purifier efficacy. One reason why indoor air quality could have been affected during the intervention period is in the case of substantial change in outdoor air quality. There were only modest changes in outdoor PM. We used ratios to help us control for the effects of differing outdoor PM over time.

Inactivity scores, non-hay fever symptoms, practical problems, and nasal symptoms all decreased in the RQLQ during the intervention period, suggesting that HEPA air purifiers might have a positive impact on life quality.

A limitation in this study is that there was no control group. It is conceivable that the concentration of $\mathrm{HDM}$ allergen decreased not because of the HEPA air purifier intervention but because of the change of seasonal climate. However, Wang et al's study ${ }^{11}$ demonstrated that HDM allergen will not change with season. Zhang et al's study ${ }^{14}$ of this matter, specific to Guangzhou, did show that the concentration of Der f1 allergen in summer was higher than in winter, however, Der $\mathrm{p} 1$ did not exhibit significant seasonal difference. Our current study demonstrated that the concentration of Der p 1 decreased after utilizing HEPA air purification, an effect not expected to be seen with seasonal changes in our province. We are not able to evaluate any potential placebo effect of the air purifier on subjective quality of life data.

In summary, HEPA air purifiers seemed to provide a favorable factor in reducing PM's concentration and HDM in the bedrooms of our subjects. Air purifiers also may effectively improve allergic rhinitis patients' quality of life.

\section{Acknowledgement}

This study is deeply thank you for financial support by GuangZhou City. Collaborative innovation of health and medical care form GuangZhou City (No.201400000002). Moreover, we truly thank you for the patients' well cooperation and the instruction from many other researchers. This study's abstract has been orally presented in 2016 APSR meeting held in Thailand on November 14, 2016 by Luo JY. Thank you all the suggestion recommended by all the expert.

Authors' attribution: Luo JY and Chen Z contributed as first author equally to this study. Guo JJ, Guo ZJ, and Lan X contributed as affiliated author to this study. Sun BQ contributed as corresponding author to this study. Moreover, Luo JY is also a submitting author to this study.

\section{References}

1. Quillen DM, Feller DB. Diagnosing rhinitis: allergic vs. nonallergic. Am Fam Physician. 2006;73:1583-90.

2. Huang SK, Zhang Q, Qiu Z, Chung KF. Mechanistic impact of outdoor air pollution on asthma and allergic diseases. J Thorac Dis. 2015;7:23-33.

3. Wang XD, Zheng M, Lou HF, Wang CS, Zhang Y, Bo MY, et al. An increased prevalence of self-reported allergic rhinitis in major Chinese cities from 2005 to 2011. Allergy. 2016;71:1170-80.

4. Gehring U, Beelen R, Eeftens M, Hoek G, de Hoogh K, de Jongste JC, et al. Particulate matter composition and respiratory health: the PIAMA Birth Cohort study. Epidemiology. 2015;26:300-9.

5. Vojta PJ, Randels SP, Stout J, Muilenberg M, Burge HA, Lynn H, et al. Effects of physical interventions on house dust mite allergen levels in carpet, bed, and upholstery dust in low-income, urban homes. Environ Health Perspect. 2001;109:815-9.

6. Chapman MD, Heymann PW, Sporik RB, Platts-Mills TA. Monitoring allergen exposure in asthma: new treatment strategies. Allergy. 1995;50 Suppl 25:S29-33.

7. Juniper EF, Guyatt GH. Development and testing of a new measure of health status for clinical trials in rhinoconjunctivitis. Clin Exp Allergy. 1991;21:77-83.

8. Seidman MD, Gurgel RK, Lin SY, Schwartz SR, Baroody FM, Bonner JR, et al. Clinical practice guideline: Allergic rhinitis. Otolaryngol Head Neck Surg. 2015;152 Suppl 1:S1-43.

9. Huang HW, Lue KH, Wong RH, Sun HL, Sheu JN, Lu KH. Distribution of allergens in children with different atopic disorders in central Taiwan. Acta Paediatr Taiwan. 2006;47:127-34.

10. Smit LA, Hooiveld M, van der Sman-de Beer F, Opstal-van Winden AW, Beekhuizen J, Wouters IM, et al. Air pollution from livestock farms, and asthma, allergic rhinitis and COPD among neighbouring residents. Occup Environ Med. 2014;71:134-40.

11. Wang Y, Xiong L, Yin X, Wang J, Zhang Q, Yu Z, et al. House dust mite allergen levels in households and correlation with allergic rhinitis symptoms. Am J Rhinol Allergy. 2014;28:193-6.

12. Agrawal SR, Kim HJ, Lee YW, Sohn JH, Lee JH, Kim YJ, et al. Effect of an air cleaner with electrostatic filter on the removal of airborne house dust mite allergens. Yonsei Med J. 2010;51:918-23.

13. Kajbafzadeh M, Brauer M, Karlen B, Carlsten C, van Eeden, Allen RW. The impacts of traffic-related and woodsmoke particulate matter on measures of cardiovascular health: a HEPA filter intervention study. Occup Environ Med. 2015;72:394-400.

14. Zhang C, Gjesing B, Lai X, Li J, Spangfort MD, Zhong N. Indoor allergen levels in Guangzhou city, southern China. Allergy. 2011;66:186-91. 